\title{
Phase Transition in Two Species Zero-Range Process
}

\author{
M. R. Evans and T. Hanney \\ School of Physics and Astronomy, University of Edinburgh, Mayfield Road, \\ Edinburgh, EH9 3JZ, UK
}

\begin{abstract}
We study a zero-range process with two species of interacting particles. We show that the steady state assumes a simple factorised form, provided the dynamics satisfy certain conditions, which we derive. The steady state exhibits a new mechanism of condensation transition wherein one species induces the condensation of the other. We study this mechanism for a specific choice of dynamics.
\end{abstract}

PACS numbers: 05.70.Fh, 02.50.Ey, 64.60.-i 


\section{Introduction}

The zero-range process has recently received widespread attention in the literature of nonequilibrium stochastic processes [1, 2, 3, 4, 5, 6, 7. There are three main reasons for this (see 3] for a review): (i) it provides a minimal model for more complex systems, (ii) the steady state has a simple factorised form, (iii) it exhibits a steady state phase transition, between a fluid and a condensate phase. The simple form of the steady state allows detailed analysis of this condensation transition. Apart from being of interest in its own right (as an example of a phase transition in an exactly solvable steady state), the transition is of particular interest because of its relation to coarsening phenomena in $1 d$ driven systems [2, 4]. Further, it has provided a general criterion to determine the existence of phase separation in driven systems with conserved density [5].

In the zero-range process, particles hop from site to site with hop rates determined by the number of particles at the departure site. Two types of condensation transition, whereby a finite fraction of the particles condense onto a single site, have been studied [3]: condensation induced by site-dependent hop rates and condensation induced by the asymptotic dependence of the hop rate on particle number at the site. The former is related to Bose-Einstein condensation whereas the latter involves a spontaneously broken symmetry.

Here, we generalise to a zero-range process with two species of particles. The hop rates of each species are determined by the numbers present of both species at the departure site. Under certain conditions which we specify, the steady state again assumes a simple factorised form, which we use to demonstrate a new mechanism of condensation transition. In this case the condensation of one species is induced by the distribution of particles of the other species.

One perspective on this two species model is as a system of particles moving on an evolving landscape - the dynamics of one particle species depend locally on how many particles of the other species are present (the landscape) and these particles too are subject to locally determined dynamics. The new condensation mechanism then is one where the condensate is induced by the landscape whose evolution is in turn coupled to the particle distribution. Such interplay between particle dynamics and an evolving landscape is of considerable interest [8, 9].

\section{Steady State}

We define a two species zero-range process as follows. Consider a chain of $L$ sites, with periodic boundary conditions, and containing $N$ particles of species $A$ and $M$ particles of species $B$. Sites, labelled $l=1, \ldots, L$, contain $n_{l}$ particles of species $A$ and $m_{l}$ particles of species $B$. Particles hop to their nearest neighbour site to the right, species $A$ with rate $u(n, m)$ and species $B$ with rate $v(n, m)$, where $n$ and $m$ are the occupation variables of the departure site. Thus the hopping rates, which depend on the number of particles of both species at the departure site, contain the interaction between species. 
The steady state for the two species model can be derived in a similar way to that for the single species zero-range process 3 . First, we define $P\left(\left\{n_{l}\right\} ;\left\{m_{l}\right\}\right)$ to be the steady state probability of finding the system in the configuration $\left(\left\{n_{l}\right\} ;\left\{m_{l}\right\}\right)$, where $\left\{n_{l}\right\}=n_{1}, \ldots, n_{L}$ and $\left\{m_{l}\right\}=m_{1}, \ldots, m_{L}$. Now, the steady state Master equation, which demands that the probability current into a particular configuration is equal to the probability current out of the same configuration, can be written

$$
\begin{aligned}
0=\sum_{l=1}^{L}\left[\left\{u \left(n_{l-1}\right.\right.\right. & \left.+1, m_{l-1}\right) P\left(\ldots, n_{l-1}+1, n_{l}-1, \ldots ; \ldots, m_{l-1}, m_{l}, \ldots\right) \\
& \left.-u\left(n_{l}, m_{l}\right) P\left(\ldots, n_{l-1}, n_{l}, \ldots ; \ldots, m_{l-1}, m_{l}, \ldots\right)\right\} \theta\left(n_{l}\right) \\
& +\left\{v\left(n_{l-1}, m_{l-1}+1\right) P\left(\ldots, n_{l-1}, n_{l}, \ldots ; \ldots, m_{l-1}+1, m_{l}-1, \ldots\right)\right. \\
& \left.\left.-v\left(n_{l}, m_{l}\right) P\left(\ldots, n_{l-1}, n_{l}, \ldots ; \ldots, m_{l-1}, m_{l}, \ldots\right)\right\} \theta\left(m_{l}\right)\right]
\end{aligned}
$$

where $\theta(x)$ is the usual Heaviside function and ensures that the site $l$ is occupied in order for a particle either to have arrived or to be able to vacate there. The first term on the r.h.s of (11) is a gain term due to an $A$ particle hopping into site $l$ from site $l-1$, the second term is a loss term due to an $A$ particle hopping out of site $l$; the third and fourth terms represent analogous processes for the $B$ particles. We seek a solution to (11) where $P\left(\left\{n_{l}\right\} ;\left\{m_{l}\right\}\right)$ is given by a product measure (i.e. a product of factors)

$$
P\left(\left\{n_{l}\right\} ;\left\{m_{l}\right\}\right)=Z_{L, N, M}^{-1} \prod_{l=1}^{L} f\left(n_{l}, m_{l}\right),
$$

where $Z_{L, N, M}$ is a normalisation. Equation (11) can be satisfied by substituting in the steady state (2), and asking that the gain and loss terms due to the dynamics of the $A$ particles cancel independently of the gain and loss terms due to the dynamics of the $B$ particles. Then, if this cancellation is achieved for each term $l$ in the sum individually, one finds that for all $l$,

$u\left(n_{l}, m_{l}\right) f\left(n_{l-1}, m_{l-1}\right) f\left(n_{l}, m_{l}\right)=u\left(n_{l-1}+1, m_{l-1}\right) f\left(n_{l-1}+1, m_{l-1}\right) f\left(n_{l}-1, m_{l}\right)$,

for all $n_{l} \neq 0$, and

$v\left(n_{l}, m_{l}\right) f\left(n_{l-1}, m_{l-1}\right) f\left(n_{l}, m_{l}\right)=v\left(n_{l-1}, m_{l-1}+1\right) f\left(n_{l-1}, m_{l-1}+1\right) f\left(n_{l}, m_{l}-1\right)$,

for all $m_{l} \neq 0$. These equations in turn imply that

$$
\begin{aligned}
& \frac{u\left(n_{l}, m_{l}\right) f\left(n_{l}, m_{l}\right)}{f\left(n_{l}-1, m_{l}\right)}=\frac{u\left(n_{l-1}+1, m_{l-1}\right) f\left(n_{l-1}+1, m_{l-1}\right)}{f\left(n_{l-1}, m_{l-1}\right)}=\text { constant }, \\
& \frac{v\left(n_{l}, m_{l}\right) f\left(n_{l}, m_{l}\right)}{f\left(n_{l}, m_{l}-1\right)}=\frac{v\left(n_{l-1}, m_{l-1}+1\right) f\left(n_{l-1}, m_{l-1}+1\right)}{f\left(n_{l-1}, m_{l-1}\right)}=\text { constant },
\end{aligned}
$$

for all $l$. Both constants are set equal to unity (they only appear as an overall factor in the normalisation). The two relations (5) and (6) imply a constraint on the choice of $u\left(n_{l}, m_{l}\right)$ and $v\left(n_{l}, m_{l}\right)$ i.e. we can use (5) and (6) to obtain two expressions for $f\left(n_{l}, m_{l}\right)$ in terms of $f\left(n_{l}-1, m_{l}-1\right)$ :

$$
f\left(n_{l}, m_{l}\right)=\frac{f\left(n_{l}-1, m_{l}-1\right)}{u\left(n_{l}, m_{l}\right) v\left(n_{l}-1, m_{l}\right)}=\frac{f\left(n_{l}-1, m_{l}-1\right)}{u\left(n_{l}, m_{l}-1\right) v\left(n_{l}, m_{l}\right)}
$$


but both of these expressions must give the same result, therefore the hopping rates are required to obey the constraint,

$$
\frac{u\left(n_{l}, m_{l}\right)}{u\left(n_{l}, m_{l}-1\right)}=\frac{v\left(n_{l}, m_{l}\right)}{v\left(n_{l}-1, m_{l}\right)},
$$

for $n_{l}, m_{l} \neq 0$. The choices of $u\left(n_{l}, 0\right)$ and $v\left(0, m_{l}\right)$ remain unconstrained.

Finally, the expression for $f\left(n_{l}, m_{l}\right)$ is obtained by iterating ([5]) and (6), hence

$$
f\left(n_{l}, m_{l}\right)=\prod_{i=1}^{n_{l}}\left[u\left(i, m_{l}\right)\right]^{-1} \prod_{j=1}^{m_{l}}[v(0, j)]^{-1},
$$

where we have set $f(0,0)=1$. Although not immediately obvious, $u(n, m)$ and $v(n, m)$ do play symmetric roles in (9), but this symmetry is obscured within the constraint (8) on the hopping rates.

Also note that we have the freedom to choose any desired form for $f\left(n_{l}, m_{l}\right)$, then by substituting this form into (15) and (6) we deduce the hopping rates which would lead to such a steady state - such rates are then guaranteed to satisfy the condition (8).

In order to look for phase transitions, it will prove useful to consider the normalisation, $Z_{L, N, M}$, defined in equation (2). This quantity plays a role analogous to the canonical partition function of equilibrium statistical mechanics. It can be written

$$
Z_{L, N, M}=\sum_{\left\{n_{l}\right\},\left\{m_{l}\right\}} \delta\left(\sum_{l=1}^{L} n_{l}-N\right) \delta\left(\sum_{l=1}^{L} m_{l}-M\right) \prod_{l=1}^{L} f\left(n_{l}, m_{l}\right),
$$

where the delta-functions ensure that we have $N$ particles of species $A$ and $M$ particles of species $B$ in the system. Using an integral representation of the delta-functions yields

$$
Z_{L, N, M}=\sum_{\left\{n_{l}\right\},\left\{m_{l}\right\}} \oint \frac{d z}{2 \pi i} \oint \frac{d y}{2 \pi i} \frac{\prod_{l=1}^{L} f\left(n_{l}, m_{l}\right) z^{n_{l}} y^{m_{l}}}{z^{N+1} y^{M+1}}
$$

which leads us naturally to define the generating function

$$
F(z, y)=\sum_{n=0}^{N} \sum_{m=0}^{M} z^{n} y^{m} f(n, m) \text {. }
$$

We now assume that in the limit $N, M, L \rightarrow \infty$ (with the particle densities held fixed), the integral in equation (11) is dominated by its saddle point. The saddle point equations, which involve the particle densities of species $A$ and $B, \rho_{A}=N / L$ and $\rho_{B}=M / L$, are found to be

$$
\rho_{A}=z \frac{\partial}{\partial z} \ln F(z, y), \quad \rho_{B}=y \frac{\partial}{\partial y} \ln F(z, y) .
$$

These equations determine $z$ and $y$. For the saddle point to be valid, $z$ and $y$ must be less than or equal to the radii of convergence of $F(z, y)$ in order that we can perform the sum (12) in the first place. We note also that because all derivatives of $F(z, y)$ are positive, the saddle point, if valid, must be unique. In the following, we will find that it is only possible to solve (13) in the allowed ranges of $z$ and $y$ for certain values of $\rho_{A}$ and $\rho_{B}$. 


\section{Phase Transition-analysis}

We now consider how a condensation transition may arise from the interaction of the two species. A general case of interest is where the hopping rates of one of the particle species depend only on the number of the particles of the other species at the site e.g. $v(n, m)=r(n)$. Then, by (6),$f(n, m)=r(n)^{-m} s(n)$, and $u(n, m)$ is determined by (5) $(r(n)$ and $s(n)$ are general functions of $n)$. In this case, the $A$ particles play the role of an evolving landscape that determines the dynamics of the $B$ particles. For simplicity, we consider the case where $r(n)=1+c /(n+1)$ and $s(n)=1$, then

$$
v(n, m)=1+\frac{c}{n+1}, \quad u(n, m)=\left(\frac{1+\frac{c}{n+1}}{1+\frac{c}{n}}\right)^{m},
$$

for all values of $n$ and $m$; $c$ is a constant. Physically, these hopping rates are such that the $B$ particles hop more slowly as the number of $A$ particles at the departure site increases, while the $A$ particles slow as the number of $B$ particles increases but they hop more quickly the more particles of the same species occupy the departure site. The generating function and its first derivatives, having performed the sum over $m$, are obtained as

$$
\begin{aligned}
& F(z, y)=\sum_{n=0}^{\infty} z^{n} \frac{1+c+n}{(1+n)(1-y)+c}, \\
& z \frac{\partial}{\partial z} F(z, y)=\sum_{n=0}^{\infty} n z^{n} \frac{1+c+n}{(1+n)(1-y)+c}, \\
& y \frac{\partial}{\partial y} F(z, y)=\sum_{n=0}^{\infty}(1+n) z^{n} \frac{y(1+c+n)}{[(1+n)(1-y)+c]^{2}} .
\end{aligned}
$$

The radii of convergence of these series are $z=1$ and $y=1$. These expressions determine $z$ and $y$, given $\rho_{A}$ and $\rho_{B}$, as prescribed by the saddle point equations (13).

One can demonstrate the existence of a phase transition in the following way. For a given $z$, both $\rho_{A}$ and $\rho_{B}$ are monotonically increasing functions of $y$. Then by analysing the saddle point equations for the four cases $y=0, z=0, y=1$, and $z=1$, the dependences of $\rho_{A}$ and $\rho_{B}$ on $y$, for fixed $z$, are obtained. These are shown in figure 11. where $0<z_{1}<z_{2}<1$. Now, if we consider a system containing a density $\rho_{A}$ of $A$ particles, then the solution of (13) requires that $z$ and $y$ lie in the range $z_{1} \leq z \leq z_{2}$ and $0 \leq y \leq 1$. In this range, $\rho_{B}$ increases monotonically from $\rho_{B}=0$, where $z=z_{2}$ and $y=0$, to its maximum value allowed by the saddle point equations, where $y=1$ and $z=z_{1}$. Therefore we can only solve the saddle point equations for $\rho_{A}$ and $\rho_{B}$ if $\rho_{B}$ is less than this ( $\rho_{A}$-dependent) maximum. When $\rho_{B}$ exceeds this maximum, the saddle point approximation is no longer valid and a phase transition ensues. The critical line is given by the values of $\rho_{A}$ and $\rho_{B}$, as a function of $z$, where $y=1$. Along this line $\rho_{A}$ and $\rho_{B}$ are related by

$$
\rho_{B}=\left(1+\rho_{A}\right) / c .
$$



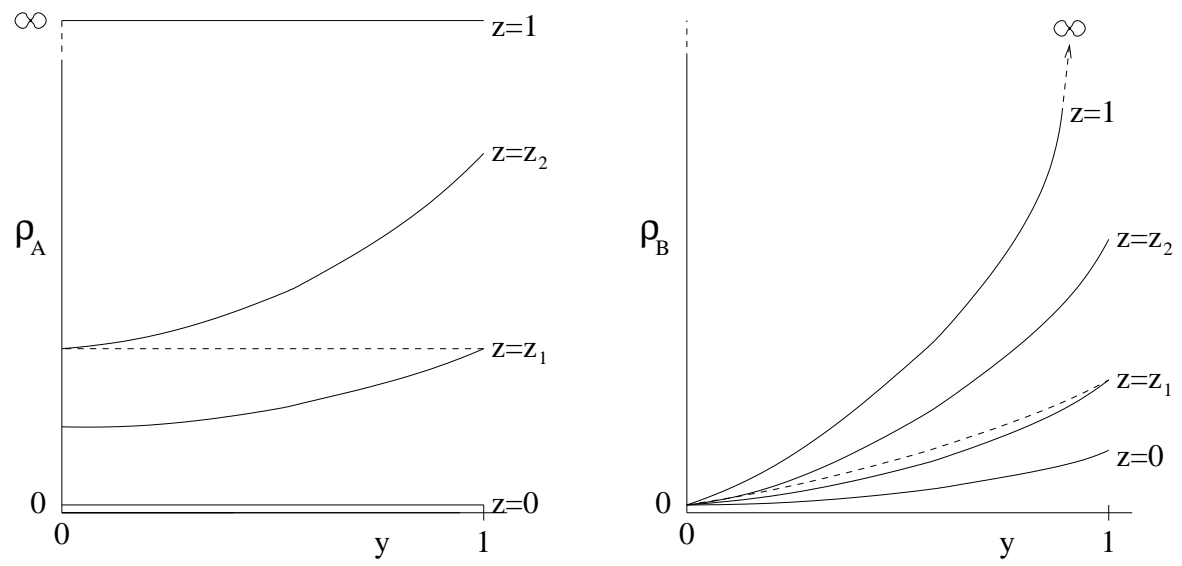

Figure 1. Schematic dependences of the particle densities $\rho_{A}$ and $\rho_{B}$ for contours of fixed $z$ and as a function of $y$. The dashed line in the right hand graph illustrates how $\rho_{B}$ varies as a function of $z$ and $y$ given that $\rho_{A}$ is fixed.

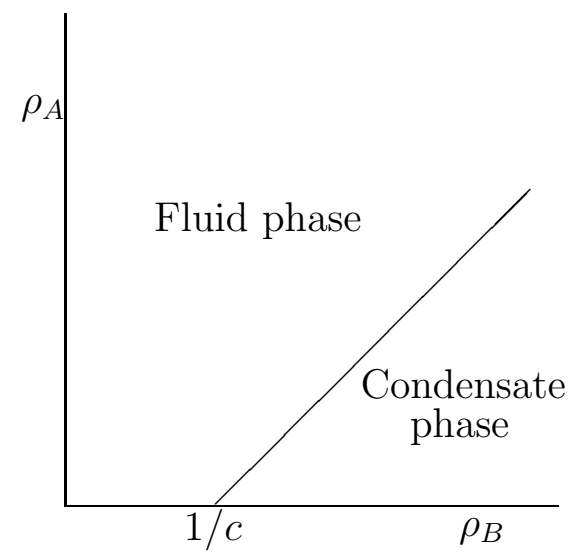

Figure 2. Phase diagram for hopping rates (14).

This is most easily seen by setting $y=1$ in (15), (16) and (17), and then spotting that one can write $y \partial F(z, y) / \partial y=[F(z, y)+z \partial F(z, y) / \partial z] / c$. Then dividing through by $F(z, y)$ yields (18). Hence we obtain the phase diagram shown in figure 2 .

We interpret this as a transition between a fluid phase, where the saddle point equations can be solved, and a condensate phase. In the condensate phase, $\rho_{B}$ exceeds a maximum critical value and the excess $B$ particles condense onto a single site. The number of $B$ particles in the condensate is proportional to the system size $L$-in the limit $N, M, L \rightarrow \infty$ it contains a finite fraction of an infinite number of particles. However, we note that for $m \rightarrow \infty, u(n, m) \rightarrow 0$ if $n$ is finite. But the current of $A$ particles must be the same across all bonds in the system as a result of continuity, and this current must be finite - the current of $A$ particles away from the condensate is finite - therefore we must have that $n \rightarrow \infty$ at the condensate in order that the current out of the condensate site is finite. For $n$ large, $u(n, m) \sim \exp \left(m / n^{2}\right)$ and this must be finite, therefore $m \sim n^{2}$ at the condensate then since $m \propto L$ here, $n \propto L^{1 / 2}$. Thus the 

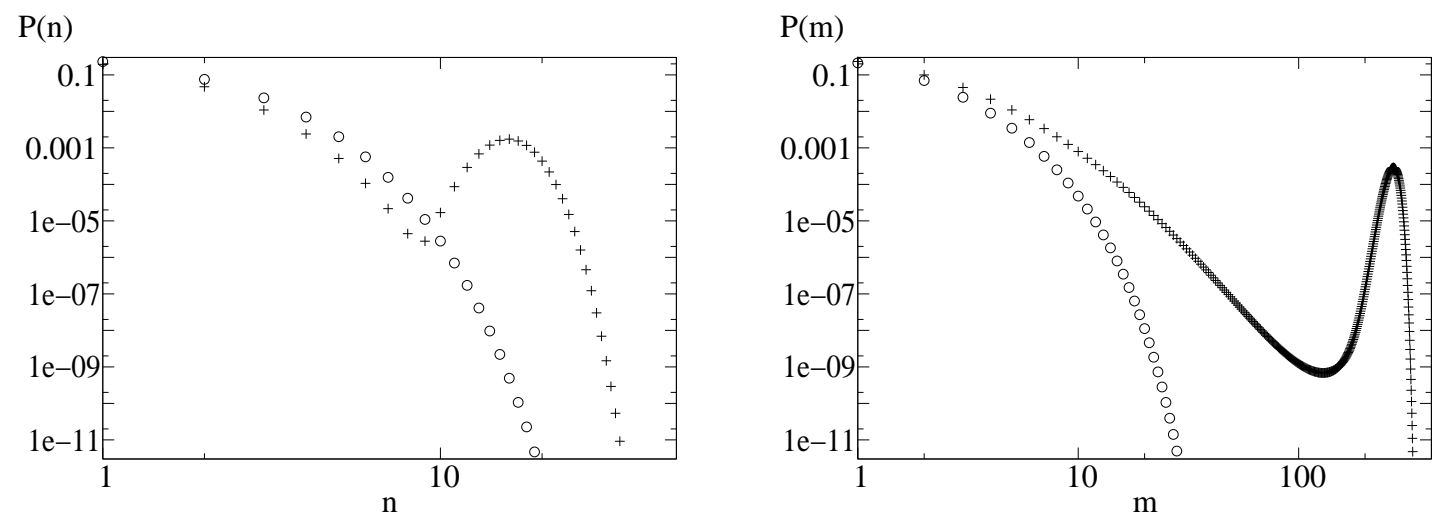

Figure 3. Log-log plot of on the left $P(n)$ vs. $n$, and on the right $P(m)$ vs. $m$, for systems of size $L=100$ and $c=2$. The circles correspond to densities $\rho_{A}=1 / 2=\rho_{B}$; the crosses correspond to densities $\rho_{A}=1 / 2$ and $\rho_{B}=7$.

condensate phase is characterised by a condensate of $B$ particles which is sustained by a 'weak' condensate of $A$ particles. This picture is consistent with numerical results: an exact recursion relation for the partition function can be obtained from (10) and is found to be

$$
Z_{L, N, M}=\sum_{n=0}^{N} \sum_{m=0}^{M} f(n, m) Z_{L-1, N-n, M-m} .
$$

This expression was iterated exactly on a computer up to systems of size $L=100$, for different values of the densities and using $c=2$. It is also straightforward to obtain an exact expression for $P(n, m)$, the probability that a site contains exactly $n$ particles of species $A$ and $m$ particles of species $B . P(n, m)$ is given by

$$
P(n, m)=Z_{L, N, M}^{-1} Z_{L-1, N-n, M-m} f(n, m) .
$$

By summing this equation over $n$ or $m$ one obtains equations for $P(n)$, the probability of finding exactly $n$ particles of species $A$ at a site, and $P(m)$, the probability of finding exactly $m$ particles of species $B$ at a site. These probabilities are plotted in figure 3 for two different values of the densities. The circles correspond to densities $\rho_{A}=1 / 2=\rho_{B}$ and the system is in the fluid phase - both $P(n)$ and $P(m)$, as expected, decay exponentially. For $\rho_{A}=1 / 2$ and $\rho_{B}=7$, represented by the crosses, the system is in the condensate phase. Within finite size effects, these results are consistent with the following picture: the condensate of $B$ particles (containing $\sim L$ particles) is sustained by a 'weak' condensate of $A$ particles (containing $\sim L^{1 / 2}$ particles); the condensates exist on a power-law distributed background of $B$ particles forming a critical fluid, and an exponentially distributed background of $A$ particles forming a normal fluid. 


\section{Conclusion}

We have shown that one may define a two species zero-range process where the steady state is described by a product measure (2) and we have derived conditions under which the product measure holds (8) . This simple form for the steady state makes the model amenable to detailed analysis. In particular, we have shown that the model undergoes a novel kind of condensation transition.

It is clearly of interest to investigate the generality of this transition with respect to the hopping rates. Since we can always construct dynamics, using (15, 6), that lead to a product measure (2) with arbitrary $f(n, m)$ there is scope within the model for further mechanisms of condensation transition [10]. Further analysis of the nature of the weak condensate is also desirable.

\section{Acknowledgments}

We thank David Mukamel for useful discussions. TH thanks EPSRC for financial support under grant GR/52497.

\section{References}

[1] F. Spitzer 1970 Adv. Math. 5246

[2] O.J. O'Loan, M.R. Evans and M.E. Cates 1998 Phys. Rev. E 581404

[3] M.R. Evans 2000 Braz. J. Phys. 3042

[4] Y. Kafri, E. Levine, D. Mukamel and J. Török 2002 J. Phys. A 35 L459

[5] Y. Kafri, E. Levine, D. Mukamel, G.M. Schütz and J. Török 2002 Phys. Rev. Lett. 89035702

[6] C. Godrèche 2003 Preprint cond-mat/0301156

[7] S. Großkinsky, G.M. Schütz and H. Spohn 2003 Preprint cond-mat/0302079

[8] B. Drossel and M. Kardar 2000 Phys. Rev. Lett. 85614

[9] D. Das and M. Barma 2000 Phys. Rev. Lett. 851602

[10] T. Hanney and M. R. Evans in preparation 